Maria José Peixoto ${ }^{1}$; Elizabete Borges ${ }^{2}$

\section{RESUMO: O SOFRIMENTO NO CONTEXTO DA DOENÇA}

Sendo o sofrimento um fenómeno biopsicossocial e espiritual ele está presente em todos os contextos associados à doença. Mas, o sofrimento não tem repercussões só para a pessoa/família mas também para os profissionais de saúde. Neste contexto propomo-nos elaborar uma pequena reflexão sobre o sofrimento das pessoas/famílias que perderam o seu bem-estar e os enfermeiros que pela inerência das suas funções estão integrados no âmago do sofrimento.

\section{PALAVRAS CHAVE: Sofrimento; Enfermeiro; Família;} Cliente

\section{ABSTRACT: SUFFERING IN THE CONTEXT OF DISEASE}

Suffering is a biopshycosossial and spiritual process and it is present in all the contexts related to disease. But, suffering has repercussions not only on the person/family but also on the health professionals. In this context we will develop a short reflection about the suffering of people who lost their welfare and the nurses, who by their inherence of the function, are integrated in the centre of suffering.

\section{KEYWORDS: Suffering; Nurse; Family; Client}

\section{INTRODUÇÃO}

O sofrimento é transversal nas várias dimensões do ser humano, circunscreve características biopsicossociais e espirituais. Etimologicamente sofrimento "denota pena ou dor que se deve suportar (latim subferre, levar debaixo) Casera (2001, p. 1043).

Classicamente fala-se no sofrimento físico, quando atribuído ao corpo e mental quando conotado com a mente. Béfécadu (1993) fala no "sofrimento do corpo - a fonte física, sofrimento nas relações interpessoais - a fonte sociocultural, sofrimento na vontade, a fonte existencial-espiritual e o sofrimento no sentimento de unidade e coerência do "eu" - a fonte psicológica" (cit por Gameiro, 1999, p. 37). Para
Biscaia (1995, p. 7) "a dor e o sofrimento humanos são atributos da pessoa já que eles pressupõem, não só uma componente neurológica, mas também a memorização e o repercutir psicológico que lhe dão uma clara implicação em comportamentos futuros".

O sofrimento é uma experiência pessoal, egocêntrica e complexa que envolve um evento intensamente negativo ou uma ameaça percebida. O sofrimento pode fazer-se acompanhar de componentes físicas, cognitivas, afectivas, sociais e espirituais o que justifica a sua complexidade (Rodgers \& Cowles, 1997).

A investigação efectuada à volta do sofrimento traduz grande proximidade conceptual, verificam-se experiências comuns e outras exclusivas que dependem não só da causa do sofrimento mas também das especificidades do indivíduo. Mas o foco do sofrimento não se deve propriamente à causa mas ao risco que representa para a identidade da pessoa (Gameiro, 1999). Apesar de não conseguirmos identificar a amplitude e o significado do sofrimento pelo qual a pessoa está a passar, normalmente conseguimos perceber quando a pessoa está a sofrer (Kahn \& Steeves, 1986). Sendo que "O ser humano é o único dotado de um sentimento intrínseco, decorrente do excesso de algo, que incomoda, perturba ou provoca insatisfação" (Berlink cit.por Malavolta, 2000).

Na cultura ocidental, em que está implícita a divisão espíritocorpo, o sofrimento é relacionado com o espírito e por isso a sua subjectividade. Mas para podermos entender o sofrimento, não podemos pensar numa lógica de espírito versus mente, pois cada ser humano é um todo, espírito e corpo (Cassel, 1992, cit. por Ribeiro, 2005). Ao analisar o sofrimento em âmbito médico, refere que "a) o sofrimento não se confina aos sintomas físicos, b) o sofrimento deriva tanto da doença como do tratamento, c) ninguém pode antecipar o que o sujeito descreverá como fonte de sofrimento" Cassel (1992, cit. por Ribeiro, 2005, p. 223).

Como o ser humano é um ser biopsicossocial e o sofrimento pode abranger todas as suas dimensões, este também pode ser desencadeado por qualquer aspecto da pessoa. O sofrimento acontece quando a pessoa se apercebe de algo que a pode colocar numa situação de risco, e termina quando consegue eliminar esse risco. Hallowell (2006) ao estudar mulheres com história familiar de cancro do ovário,

\footnotetext{
Professora Coordenadora - Escola Superior de Enfermagem do Porto, mariajose@esenf.pt

2 Professora Adjunta - Escola Superior de Enfermagem do Porto, elizabete@esenf.pt Submetido em 16-06-2011. Aceite em 16-12-2011.

Citação: Peixoto, M. J.; Borges, E. (2011). O sofrimento no contexto da doença. Revista Portuguesa de Enfermagem de Saúde Mental. 6, 36-39.
} 
constatou que estas percebem o risco da doença pela experiência anterior da doença e morte e encaram o futuro com sofrimento para elas e para os outros. Doentes com o diagnóstico de uma doença crónica apresentam sofrimento devido a perda de autonomia, diminuição da auto-estima, desânimo (Morita et al., 2000) e percepção da perda da integridade (Rodgers \& Cowles, 1997).

Visto que no sofrimento está implícita a subjectividade, é óbvio que este não apresenta similaridade na sua expressão, não sendo através desta que o alcançámos e que o podemos identificar. Mas também a habilidade para enfrentar o sofrimento é diferente e pessoal. Situações há em que o ser humano descobre como viver com o seu sofrimento, não será o caso das pessoas que têm dores crónicas? Há situações que se apresentam consensuais, no que se refere a serem as geradoras de provocar sofrimento nas pessoas, como o caso da morte, doença... Mas, outras há que não são tão lineares. Contudo esse sofrimento também deve ser considerado e não desvalorizado perante a pessoa que o está a sentir (Ribeiro, 2005). Por vezes acontece que as pessoas doentes tentam proteger-se a si e aos outros, escondendo o quanto estão a sofrer e este é um comportamento causador de mais sofrimento ainda (Flaming, 1995). Numa perspectiva religiosa, o sofrimento pode gerar incerteza, mas também pode sustentar a confiança (Jaspard, 2004). Nesta óptica o sofrimento não deve ser interpretado como um sinal de infortúnio, mas sim como um guia de novas expectativas, visto possibilitar ver as situações com outra clareza. Assim a pessoa ao assumilo como uma manifestação de redenção está a proporcionar mais qualidade à vida (Pinto, 1996).

Também Frankl (1984), ao analisar o sofrimento humano, defende que a dor e o sofrimento, de uma forma geral, são indispensáveis ao desenvolvimento humano.

\section{SOFRIMENTO E FAMÍLIA CUIDADORA}

Ao longo do tempo tem existido alguma preocupação em estudar este fenómeno, mas, mais no contexto do sofrimento do utente e da dor, e não, na perspectiva da família cuidadora. Assiste-se hoje a um incremento de famílias cuidadoras, e perante esta realidade parece lícito então questionar se essas famílias não passam por uma transição de vivencias durante períodos de sofrimento. Vários estudos (Martins, 2006; Fernandes et al. 2002; Gunnell et al. 2000) apontam para que nas famílias cuidadoras essencialmente, o familiar cuidador manifesta elevada sobrecarga física, emocional e social. A doença de um dos elementos da família vai implicar ruptura no quotidiano do próprio e da família, levando a alterações das rotinas diárias e por vezes à instauração de uma crise familiar. O impacto do diagnóstico de uma doença não só se repercute na pessoa, mas também na família (Hinds, 1992). "O doente sofre e também faz sofrer" (Bernardo, 1995, p.3). O aparecimento no seio familiar de alguém que vai precisar de ajuda para a realização das actividades diárias, que normalmente resolve sozinho, vai acarretar alterações substanciais na família (Roca et al. 1999). A doença crónica, mais do que a aguda, significa não só para a pessoa individual mas para a unidade familiar uma ameaça (Bull et al 1997). Uma doença grave traz uma série de imposições e de alterações para a família que colidem com a forma de viver o seu dia a dia. A doença é uma das causas de sofrimento (Sebastião, 1995) e quer seja temporária ou prolongada, em qualquer etapa do ciclo vital acarreta consigo diferentes níveis de stresse (Hanson, 2005), o qual é um dos factores responsáveis pelo sofrimento (Borges, 2004).

\section{O ENFERMEIRO E O SOFRIMENTO}

Flaming (1995) estudou a percepção das enfermeiras em relação ao sofrimento dos utentes, visto aquelas pertencerem a um grupo de pessoas que no seu dia a dia de trabalho contactam constantemente com diferentes situações. Assim verificou que as enfermeiras na sua prática diária identificaram o sofrimento existencial, o psicológico e o físico. O existencial, contempla o aspecto espiritual e o desespero pela situação, acontecendo quando a pessoa sente que essa dimensão da sua vida perdeu o sentido. $O$ sofrimento psicológico sucede frequentemente quando há um acometimento à parte psicológica da pessoa, que se traduz pela incompreensão. Esta não acredita no que está a acontecer, sente-se presa e não compreende a situação. O sofrimento físico associa-se à dor corporal, podendo manifestar-se de forma inflexível e exigente.

Quando a pessoa aceita a existência do sofrimento torna-se mais fácil chegar a ela de forma a minimizá-lo, podendo ser atenuado caso o utente tenha coragem de o exprimir e de o encarar. Os profissionais de enfermagem pela sua experiência normalmente conseguem identificar a existência de sofrimento na pessoa. Os enfermeiros consideram que a relação de confiança que se estabelece entre eles e o utente, baseada na abertura e sinceridade, é crucial para conseguirem intervir a esse nível. É imprescindível que a pessoa nunca se sinta abandonada, que seja respeitada, pois esta tem uma experiência de vida que pode ajudar a minimizar o sofrimento (Lindholm \& Eriksson, 1993). É importante que os profissionais de enfermagem percebam a energia que é necessária para que a pessoa expresse os seus sentimentos. Este processo pode utilizar muita da energia quer da pessoa, quer do profissional. Os profissionais podem identificar o tipo de sofrimento que está consciente ou inconscientemente a ser manifestado pelo utente, levando a que este entenda quais os tipos de sofrimento existente. Por vezes os utentes usam o sofrimento como um mecanismo de defesa, os profissionais devem entender esse comportamento e considerá-lo. Os enfermeiros devem respeitar o espaço da pessoa, na medida em que esta pode sentir necessidade de estar só (Flaming, 1995). 
O enfermeiro por vezes tenta aliviar o sofrimento respondendo de imediato às necessidades e ajudando o utente a resolver os seus problemas. Contudo devem ter sempre presente que nada deve ser imposto e que o caminho deve ser escolhido com a pessoa. Para isso é importante encontrar as razões do sofrimento conseguindo que os utentes o façam conjuntamente com eles. É também importante que o utente crie uma relação com uma pessoa que seja significativa para si. É essencial que tenha coragem de encarar o sofrimento e caminhar em frente (Lindholm \& Eriksson, 1993). Mock et al. (2005) estudaram o sofrimento de um grupo de enfermeiras que tinham síndrome respiratório e concluíram que estas se tornaram mais "ricas" nas relações com as pessoas quer profissionalmente quer mesmo na sua vida pessoal. Passaram a ver o mundo na perspectiva dos utentes e consciencializaram-se da importância de ouvirem a pessoa e a família, respeitando-os.

Mas, é inevitável que quando se fala em sofrimento ligado à doença, não só se pense na pessoa doente e família, mas também nos profissionais de saúde nomeadamente nos enfermeiros. Estes são confrontados na sua prática diária com diversas formas de sofrimento humano (Kahn \& Steeves, 1986). Jaspard (2004) estudou como estes profissionais convivem constantemente com o sofrimento, considerando a sua orientação religiosa. Encontrou diferenças na forma como estes se referiam ao sofrimento, atendendo à óptica religiosa ou não. Assim as enfermeiras que se suportavam numa religião responderem às questões de forma distinta, enquanto as outras pelo contrário, tinham respostas idênticas. Os enfermeiros por vezes acabam por sofrer com a pessoa em sofrimento (Lindholm \& Eriksson, 1993).

Lemos, Cruz e Batomé (2002) quis estudar um grupo de profissionais de saúde nomeadamente enfermeiros, técnicos e auxiliares de enfermagem pois considerou-os como sendo um grupo de trabalhadores, que constantemente, não só assistem, mas também estão integrados no cenário onde o sofrimento é detentor do papel principal. Mesmo assim estes continuam a trabalhar, tolerando o sofrimento, por isso pareceu-lhe relevante perceber como é que estes profissionais gerem as adversidades. Para isso seleccionou como participantes os que trabalhavam em centros cirúrgicos de adultos, urgência, clínica e ambulatório de onco-hematologia. A caracterização dos processos de trabalho apontou para a presença de cargas físicas e psíquicas. Constatou a existência de sofrimento, causada por agentes relacionados com a organização do trabalho, nomeadamente escassez de recursos materiais e humanos que impossibilitavam a prestação de cuidados com qualidade. Mas visto que para os profissionais de saúde é inevitável a prestação de cuidados, Lemos, Cruz e Batomé (2002, p. 408) constatou a existência de mecanismos de defesa que por vezes são utilizados sem os próprios terem consciência do facto, tais como a "negação, sublimação, e a banalização do sofrimento, da assistência e até das informações prestadas aos pacientes e seus familiares". Borges (2004) ao estudar o sofrimento dos enfermeiros em
Pediatria constatou que para estes profissionais a convivência com o sofrimento das crianças, pais e familiares era factor desencadeante de sofrimento para os próprios. Este pode ser exacerbado pela "situação clínica da criança, a relação de proximidade com a criança e os pais e os comportamentos adoptados pelos pais. Não podemos deixar de referir os indicadores associados ao momento da morte, como o dia, as circunstâncias e as condições que envolvem este acontecimento" (Borges, 2004, p. 129). Confrontados com o sofrimento das crianças os enfermeiros podem desenvolver dois tipos de comportamento, ou a aproximação à criança e aos pais ou o distanciamento. Os enfermeiros em situações mais "pesadas" adoptam estratégias de coping centradas na emoção e no problema. As emoções podem ser geridas a nível individual ou de organização, passando por "apoio psicológico, reestruturação física dos serviços, a formação, a valorização do trabalho dos enfermeiros e a rotatividade pelos serviços" (Borges, 2004, p. 130). Nas situações de stress foram identificadas como respostas "Negação; Raiva e Frustração; Distress e Saúde; Culpabilidade; Resposta Fisiológica e Emocionalidade Disfórica" (Borges, 2004, p. 130). "O modo como cada enfermeiro enfrenta situações de crise, nomeadamente o sofrimento... reflecte a sua individualidade enquanto ser único e a rede de ligações enquanto ser relacional” (Borges, 2004, p. 43).

\section{CONSIDERAÇÕES FINAIS}

O sofrimento integra a vida do ser humano e está presente em todas as situações que impliquem cuidados. Este não pode ser reduzido a apenas um sentimento ou uma dor; assistimos a sofrimento na vida e na morte, este é único, com intensidade variável podendo ser aliviado mas não suprimido. Existe sofrimento com doença sendo que as manifestações podem ser de tal forma diversas que se torne quase impossível reconhecê-las (Lindholm \& Eriksson, 1993). O sofrer do ser humano não traz originalidade, mas pode ser considerado um desafio nos nossos dias, pois assiste-se hoje a novas formas de sofrimento tais como pressões sociopolíticas, desemprego, indefinição do futuro. È premente entender todos estes novos processos que desencadeiam o sofrimento (Malavolta, 2000). Entendê-lo é fundamental para os profissionais da saúde, não só o fenómeno em si mas as estratégias de o diminuir. Mas, visto que este não acontece somente ao utente e família mas também aos profissionais de saúde, torna-se premente que sejam criadas condições para aliviar senão eliminá-lo dos profissionais. É imprescindível que os profissionais mantenham boa saúde de forma a poderem ter um bom nível de bem-estar e consequentemente prestarem cuidados de qualidade aos utentes/famílias.

Estamos a falar em competências pessoais e de relação, recursos humanos e materiais, programas de formação, de reflexão acerca do tema, de gestão de emoções. (Borges, 2004). Rushton et al. (2006) ao implementar e avaliar um programa de intervenção num serviço de cuidados paliativos 
verificou uma diminuição do sofrimento nos profissionais de saúde. Tal como refere Mclntyre (1999, p. 24) "o sofrimento acarreta alguma forma de desregulação emocional. (...) Torna-se assim crucial que os profissionais de saúde estejam preparados para reconhecer e lidar com as emoções do doente e as suas próprias emoções (...)".

\section{REFERÊNCIAS BIBLIOGRÁFICAS}

Bernardo, F. (1995). Ser e Estar Doente. Divulgação, 34, 3-6.

Biscaia, J. (1995). Sofrimento e dor humana. Cadernos de Bioética, 9, 7-13.

Borges, E. (2006). O Sofrimento dos enfermeiros em pediatria. In P. Marques, J. C.

Bull, M. J.; Jervis, M. A. \& Lori L. (1997). Strategies used by chronically ill older women and their caregiving daughters in managing posthospital care. Journal of Advanced Nursing, 25 (3), 541-547.

Carvalho, P. Sousa, E. Borges, \& S. Cruz (Eds.), Rumo ao Conhecimento em Enfermagem (pp. 248-340). Porto: Escola Superior de Enfermagem de S. João.

Casera, D. (2001). Sofrimento. In: Leone, S., Dicionário de Bioética. Vila Nova de Gaia: Editorial Perpétuo Socorro, 2001.

Fernandes, M. C., Pereira, M. L. S., Ferreira, M. Az., Machado, R. F. \& Martins, T. (2002). Sobrecarga física, emocional e social nos cuidadores informais de doentes com AVC. Sinais Vitais, 43, 31-35.

Flaming, D. (1995). Patiente suffering: a taxonomy from the nurse's perspective. Journal of Advanced Nursing, 22 (6), 1120-1127.

Frankl, V. (1984). Em Busca de Sentido. Obtido em Março de 2010 através de: http://www.endireitar.org/site/artigos/ livros/193-a-mensagem-de-viktor-frankl

Gameiro, M. H. (1999). Sofrimento na Doença. Coimbra: Quarteto.

GunnelL, D., Coast, J., Richards, S. H., Peters, T. J., Pounsfunf, J. C. \& Danlow, M. A. (2000). How great a burden does early discharge to hospital-at-home impose carers? A randomized controlled trial. Age and Ageing, 29 (2), 137-142.

Hallowell, N. (2006). Varieties of suffering: living with the risk of ovarian câncer. Health Risk \& Society, 8, 9-16.

Hanson, S. M. H. (2001). Enfermagem de Cuidados de Saúde à Família - Teoria, Prática e Investigação. Loures: Lusociência - Edições Técnicas e Científicas, Lda.
Hinds, C. (1992). Suffering: a relatively unexplored phenomenon among family caregivers of non-institutionalized patients with câncer. Journal of Advanced Nursing, 17 (8), 918-925.

Jaspard, J. M. (2004). Significação Religiosa do Sofrimento e Posição Psicológica na Fé. Psicologia USP, 15 (3), 191-212.

Kahn, D. L. \& Steeves, R. H. (1986). The Experience of Suffering: Conceptual Clarification and Theorical Definition. Journal of Advanced Nursing, 11(6), 623-631.

Lemos, J. C., Cruz, R. M. \& Batomé, S. P. (2002). Sofrimento psíquico e trabalho de profissionais de enfermagem. Estudos de Psicologia, 7 (2), 407-409.

Lindholm, L. \& Eriksson, K. (1993). To Understand and alleviate suffering in a caring culture. Journal of Advanced Nursing, 18 (9), 1354-1361.

Malavolta, A. (2002). As novas formas de sofrimento. Obtido em Março de 2007 através de: http://www.unicamp.br/ unicamp/unicamp_hoje/ju/out2000/pagina8e9-Jul55.html

Martins, T. (2006). Acidente Vascular Cerebral. Qualidade de Vida e Bem-Estar dos Doentes e Familiares Cuidadores. Coimbra: Formação e Saúde, Lda.

Mcintyre, T. M. (1995).Abordagens psicológicas do sofrimento do doente. In Mcintyre, T. M.; Carmo, V.C., O Sofrimento do Doente. Leituras Multidisciplinares (pp 17-31). Braga: Associação dos Psicólogos Portugueses.

Morita, T., Tsunoda, J., Inoue, S. \& Chihara, S. (2000). An exoloratory factor analysis of existencial suffering in Japanese terminally ill câncer patients. Psycho-Oncolgy, 9, 164-168.

Mock, E., Chung, B. P., Chung, J. W. \& Wong, T. K. (2005). An exploratory Study of nurses suffering from severe acute respiratory syndrome. International Journal of Nursing Practice, 11(4), 150-160.

Pinto, P. V. F. (1996). Sofrimento e o sentido da vida. In Servir. 44(), p. 226-231.

Ribeiro, J. L. P. (2005). Introdução à Psicologia da Saúde. Coimbra: Quarteto.

Roca, M. Ubeda, I., Garcia, L., Fuentelsaz, C. \& Verdura, T. (1999). Se cuida a la família de los enfermos crónicos. Rol Enf, 22 (1), 75-78.

Rodgers, B. L. \& Cowles, K. V. (1997) - A conceptual foundation for human suffering in nursing care and research. Journal of Advanced Nursing, 25 (5), 1048-1053.

Sebastião, L. (1995). A pedagogia da dor e do sofrimento. Cadernos de Bioética, 9, 41-45. 